\title{
Corrigendum: The refined structure of nascent HDL reveals a key functional domain for particle maturation and dysfunction
}

Zhiping Wu, Matthew A Wagner, Lemin Zheng, John S Parks, Jacinto M Shy III, Jonathan D Smith, Valentin Gogonea \& Stanley L Hazen Nat. Struct. \& Mol. Bio. 14, 861-868 (2007); published online 5 August 2007; corrected after print 27 February 2008

In the version of this article initially published, Figures $2 \mathrm{c}, \mathrm{d}$,e and 5 showed an incorrect model for discoidal HDL, with the apoA1 molecules in a clockwise orientation, in contrast with their counterclockwise orientation in the final model described in the text. The correct model is deposited under the same accession number, PM0074956, at http://mi.caspur.it/PMDB/main.php. The error has been corrected in the PDF and HTML versions of the article.

\section{Figure 2}

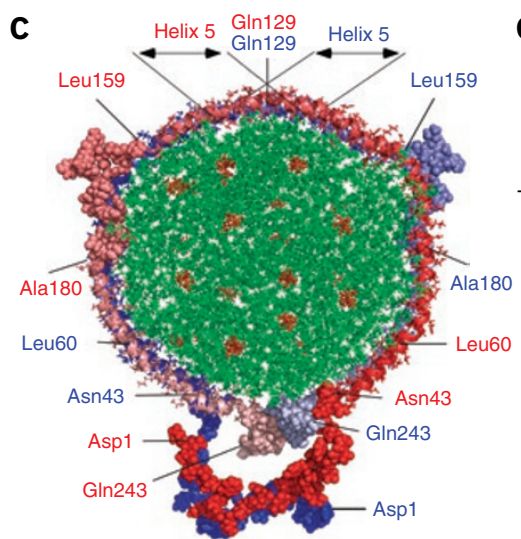

d

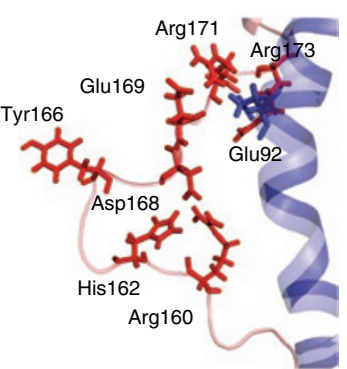

Figure 5

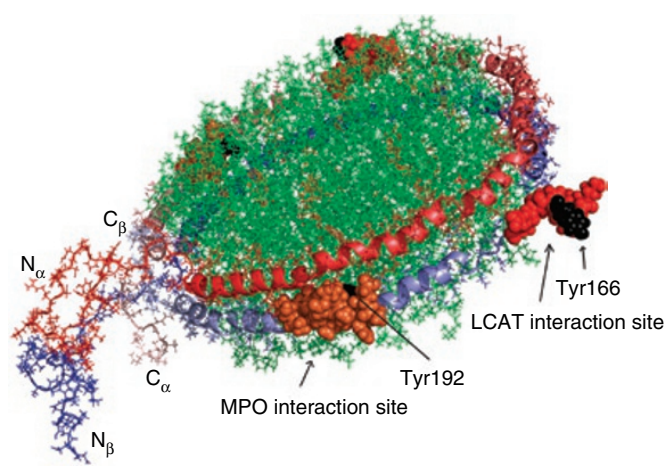

e

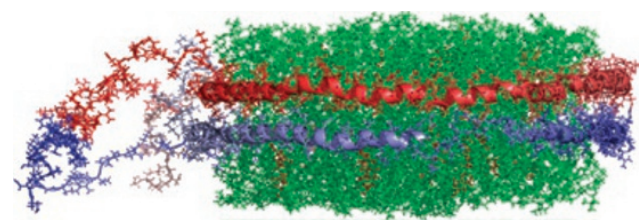

